\title{
ATIVIDADES ALELOPÁTICAS DE NIM SOBRE O CRESCIMENTO DE SORGO, ALFACE E PICÃO-PRETO
}

\author{
Allelopathic activities of nim on the growth of sorghum, lettuce and Bidens pilosa
}

\author{
André Cabral França', Itamar Ferreira de Souza ${ }^{2}$, Cláudio Costa dos Santos ${ }^{3}$, \\ Eliane Queiroga de Oliveira ${ }^{4}$, Cristiano Martinotto ${ }^{5}$
}

\begin{abstract}
RESUMO
A busca de alternativas para os herbicidas sintéticos, como os produtos naturais provenientes de plantas, pode acrescentar mais um elemento no manejo das plantas daninhas das culturas. Neste trabalho objetivou-se verificar o potencial alelopático do nim (Azadirachta indica A. Juss.) sobre o crescimento de plântulas de sorgo, alface e picão-preto. Extratos metanólico, aquoso e hexanólico, nas concentrações de 10, 5 e 2,5\% (p/v) de folhas de nim, foram usados para irrigar sementes das plantas teste, em caixas gerbox, permanecendo em incubadora, tipo BOD. O percentual de germinação de sementes de alface e picão-preto irrigadas com extrato aquoso e metanólico, respectivamente, foram os mais prejudiciais ao desenvolvimento das plântulas; para o sorgo independente do extrator utilizado, o aumento da concentração causou redução do percentual de germinação. O extrato aquoso causou maior efeito detrimental sobre o índice de velocidade de germinação para as três espécies estudadas. Para o sorgo, independente da concentração avaliada, o extrato aquoso foi o mais prejudicial, diferenciando dos demais extratores, com médias de 8,00; 12,986 e 14,68, para os extratores água destilada, hexanol e metanol, respectivamente. Quando se analisou o acúmulo de biomassa para plantas de picão-preto e sorgo, em todos os extratos utilizados, pôde-se verificar que o acúmulo de biomassa foi inversamente proporcional à concentração de nim aplicada.
\end{abstract}

Termos para indexação: Alelopatia, Azadirachta indica, inibição.

\begin{abstract}
The search for alternatives for the synthetic herbicides, as the natural products from plants, may increase one more element to the handling of the harmful plants of the cultures. The aim of this work was to verify the allelopathic effects of neem (Azadirachta indica A. Juss.) upon sorghum, lettuce, and Bidens pilosa L. (beggustick) seeds germination and seedlings growth. Aqueous, methanolic, and hexanolic extracts, in the concentrations of 10,5 and 2,5\% (w/v) of nim leaves, were used to irrigate seeds of the plant test, in gerbox boxes, staying in incubator, type BOD. Germination percentages of lettuce seeds and beggustick irrigated with aqueous and methanolic extracts, respectively, were the most harmful to the development of the plants; for sorghum, independently of the used extractor, the increase of the concentration caused reduction of the germination percentages. The aqueous extract caused larger detrimental effect on the index of germination speed for the three studied species. For the sorghum, independently of the appraised concentration, the aqueous extract was the most harmful, differing from the other extractors, with averages of 8.00; 12.986 and 14.68 , for the extractors distilled water, hexanol and methanol, respectively. When the biomass accumulation was analyzed for beggustick plants and sorghum, in all used extracts, it could be verified that the biomass accumulation was inversely proportional to the concentration of applied neem.
\end{abstract}

Index terms: Allelopathic, Azadirachta indica, inhibition.

(Recebido em 18 de maio de 2006 e aprovado em 3 de maio de 2007)

\section{INTRODUÇÃO}

A alelopatia pode ser definida como um processo pelo qual, produtos do metabolismo secundário de um determinado vegetal são liberados, impedindo ou estimulando a germinação e o desenvolvimento de outras plantas relativamente próximas, através da liberação de substâncias pelas partes aéreas, subterrâneas ou pela decomposição do material vegetal (LORENZI, 2000).

Um dos principais estudos do uso da alelopatia é a pesquisa sobre produtos naturais para o controle de plantas daninhas, visando a redução do uso de herbicidas

\footnotetext{
'Engenheiro Agrônomo, Mestre - Departamento de Agricultura/DAG - Universidade Federal de Lavras/UFLA - Cx. P. 3037 - $37200-000$ - Lavras, MG cabralfranca@yahoo.com.br

Engenheiro Agrônomo, Ph. D., Professor Adjunto - Departamento de Agricultura/DAG - Universidade Federal de Lavras/UFLA - Cx. P. 3037 - $37200-000$ Lavras, MG - itamarfs@ufla.br

${ }^{3}$ Engenheiro Agrônomo, Mestre, Professor, Pesquisador - EPAMIG/Pitangui - Rua João Batista Silva, 75 - Chapadão - Cx. P. 43 - $35650-000$ - Pitangui, MG - csantoscosta@bol.com.br

${ }^{4}$ Bióloga, Mestre - Departamento de Agricultura/DAG - Universidade Federal de Lavras/UFLA - Cx. P.3037 - $37200-000$ - Lavras, MG eliqueroga04@yahoo.com.br

${ }^{5}$ Doutor - Departamento de Biologia/DBI - Universidade Federal de Lavras/UFLA - Cx. P. 3037 - 37200-000 - Lavras, MG - cmartinotto@yahoo.com.br
} 
sintéticos, preservando o meio ambiente, assim contribuindo para agricultura sustentável (CARVALHO et al., 2002; FERREIRA \& ÁQUILA, 2000; SANTOS et al., 2004).

O produto secundário liberado pelas plantas é chamado de aleloquímico, onde hoje com estudos cada vez mais avançados da alelopatia sabe-se que tem atividades específicas na planta e no ambiente, como atividades repelentes, seletividade aos herbicidas, alelopatia e outros (NIEMEYER, 1988). Em destaque podese citar cinmethylin e sorgoleone como aleloquímicos com potencialidades de herbicidas (SOUZA et al., 1999).

Tradicionalmente, para determinação do potencial alelopático de uma planta, tem-se recorrido inicialmente à técnica dos extratos aquosos e orgânicos. Realizada em laboratório e casa de vegetação, esta técnica é considerada a mais simples e usual, fundamentada na capacidade de melhor isolar o efeito alelopático de outras interferências (GOMIDE, 1993).

O solvente mais utilizado nas extrações é a água destilada, seguido por solventes orgânicos de vários graus de polaridade. $\mathrm{O}$ emprego de extrato aquoso em testes alelopáticos tem como objetivo simular o que acontece na natureza (MEDEIROS, 1989).

O nim (Azadirachta indica A. Juss.) é nativo de Bruma, sudoeste da Ásia, tida como uma planta cosmopolita e uma árvore considerada importante economicamente (AERTS \& MORDUE, 1997). Utilizada a mais de 2000 anos no controle de pragas, nematóides, fungos e bactérias no leste asiático, como fertilizantes e na alimentação animal proporcionando um esterco de excelente qualidade e como planta com propriedades medicinais (anti-séptico, curativo e vermífugo) (SCHMUTTERER, 1990).

A espécie se tornou bem conhecida nos últimos trinta anos quando seu principal composto, a azadiractina, foi isolado. A molécula da azadiractina é muito complexa e ainda não se tem conhecimento de sua síntese. Este composto um tetranortriterpenóide, é comprovadamente eficiente no combate a muitos insetos-pragas dos mais diversos tipos de culturas (SCHMUTTERER, 1990).

Como nos herbicidas, a busca de sucedâneos para os inseticidas sistêmicos, como os produtos naturais provenientes de plantas, pode ser uma alternativa no manejo das pragas chaves das culturas (MAREDIA et al., 1992).

Cientes da grande importância das interações alelopáticas entre plantas, neste trabalho procurou-se verificar, por meio de testes "in vivo", o potencial alelopático do nim (Azadirachta indica A. Juss.) sobre crescimento de plântulas de sorgo (Sorghum bicolor (L.) Moench), alface (Lactuca sativa L.) e picão-preto (Bidens pilosa L.).

\section{MATERIAL E MÉTODOS}

O bioensaio foi conduzido no Departamento de Agricultura, da Universidade Federal de Lavras, MG, em delineamento inteiramente casualizado, com 5 repetições, durante o mês de agosto de 2005. Os tratamentos foram constituídos por 3 extratores (água destilada, metanol e hexano) de polaridades diferenciadas e 4 concentrações (10; $5 ; 2,5$ e $0 \%$ peso/volume) de folhas de nim. O tratamento $0 \%$ foi constituído apenas de água destilada, tido como testemunha.

Os extratos foram preparados com folhas da planta do nim que se encontrava em estádio vegetativo. Após serem colhidas, as folhas foram secas em estufa de circulação forçada de ar à $55^{\circ} \mathrm{C}$, até atingir o peso constante e picadas. Tomaram-se quantidades deste material (50 g para $500 \mathrm{ml}$ de cada solvente), permanecendo em repouso por 24 horas em bécker prontamente tampado e envolto com papel alumínio (evitando a fotodegradação), formando a solução estoque, de concentração $10 \%$ (p/v), no qual foi diluída com os solventes para a formação das demais concentrações utilizadas. Após este período, filtraram-se os extratos através de algodão hidrófilo.

$\mathrm{O}$ teste foi realizado em caixas gerbox transparentes $(11 \mathrm{X} 11 \mathrm{~cm})$ que constituíam as parcelas experimentais, sendo forradas com 2 folhas papel mata borrão, previamente autoclavadas à $120^{\circ} \mathrm{C}$ por uma hora, e umedecidas com volume de solução na quantidade de 2,5 vezes o peso dos papéis. Para o extrator inorgânico (água destilada), a solução foi aplicada diretamente sobre os papéis, e para os extratos orgânicos (metanol e hexano) depois de aplicados, os gerbox permaneceram abertos em temperatura ambiente, por 20 minutos, para completa evaporação dos reagentes utilizados e posteriormente irrigados com água destilada para a semeadura das espécies.

Os gerbox foram semeados com 20 sementes de cada espécie (sorgo - cv. BR 601; alface - cv. Regina de Verão; e picão-preto), as quais permaneceram em câmara de incubação, com temperatura de $20^{\circ} \mathrm{C} \pm 2$ para o picãopreto e alface e, $30^{\circ} \mathrm{C} \pm 2$ para o sorgo, com fotoperíodo de 12 horas de luz (BRASIL, 1992).

As sementes germinadas foram contadas diariamente, iniciando no dia após a semeadura das espécies e finalizando ao $6^{\circ}$ dia após o início do experimento. Foram calculados os Índices de Velocidade de Germinação (IVG) e percentual de germinação, utilizando-se como critério o aparecimento da radícula de 
comprimento maior que $50 \%$ do tamanho da semente para evitar falsa germinação por expansão do embrião com a embebição (LABOURIAU, 1983). O IVG foi calculado de acordo com metodologia de Maguire (1962).

Ao $7^{\circ}$ dia após a semeadura, as plantas foram levadas à estufa de circulação forçada de ar $\left(55^{\circ} \mathrm{C}\right)$, até peso constante, onde foi verificado o acúmulo de biomassa das plântulas.

Aos dados foram aplicados análise de variância, a comparação das médias foi realizada pelo teste de ScottKnott, ao nível de 5\% de probabilidade e análise de regressão, utilizando-se o software Sisvar.

\section{RESULTADOS E DISCUSSÃO}

Foram observadas diferenças significativas na interação envolvendo as concentrações e os extratores para percentual de germinação e índice de velocidade de germinação de alface e picão-preto, e para o acúmulo de biomassa do sorgo e do picão-preto, onde foram desdobradas as concentrações do nim dentro de cada extrator, sendo ajustada equação de regressão.

De acordo com a Figura 1, pode-se observar que ao se utilizar o extrato aquoso na concentração $(10 \% \mathrm{p} / \mathrm{v})$, obteve-se o menor percentual de germinação de sementes de alface com média aproximada de $80 \%$. Quando se utilizou-se a menor concentração $(2,5 \%$ p/v) de nim, podese observar que os extratos orgânicos (metanol e hexano) foram os que apresentaram os maiores percentuais de plântulas germinadas, diferenciando-se do extrator inorgânico (água), onde este obteve a média de $97 \%$ de germinação de plântulas de alface. Diferencia-se do trabalho apresentado por Sindhu et al. (2005) que, utilizando

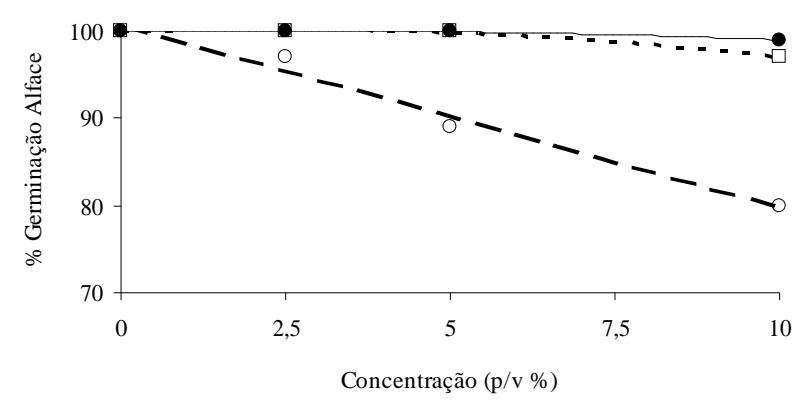

-__ _- _ Aquoso - $\mathrm{y}=-2,08 \mathrm{x}+100,6 ; \mathrm{R}^{2}=98,17$

- - - - Hexano - y = - $0,0545 \mathrm{x}^{2}+0,2564 \mathrm{x}+99,918 ; \mathrm{R}^{2}=98,79$

Metanol - $\mathrm{y}=-0,0182 \mathrm{x}^{2}+0,0855 \mathrm{x}+99,973 ; \mathrm{R}^{2}=98,79$

Figura 1 - Percentual de germinação de plântulas de alface, submetidas à concentrações de extratos de nim $(\mathrm{p} / \mathrm{v})$, utilizando água destilada, hexano e metanol como extratores. UFLA, Lavras - MG, 2007 extrato aquoso, na concentração de $10 \%$ de folhas verdes de nim, obtiveram estímulo na germinação de sementes de Amaranthus viridis L. e Parthenium hysterophorus L.

Pode-se observar que para o percentual de germinação de picão-preto (Figura 2), o extrato metanólico proporcionou maior redução, atingindo média de $46 \%$, na concentração de $2,5 \%$ (p/v). Em todas as concentrações testadas, observou-se que, quando foi utilizado este extrator, houve um maior aparecimento de plântulas anormais e com radículas necrosadas desta espécie, não sendo quantificado. O extrato hexanólico foi o que proporcionou maiores percentuais de germinação, atingindo médias de 59, 48 e $46 \%$ para as concentrações de nim 2,5; 5 e $10 \%(\mathrm{p} / \mathrm{v})$, respectivamente e confirmado com Blum (1999), que afirma que a emergência da plântula e seu crescimento inicial são as fases mais sensíveis da ontogênese do indivíduo, ou seja, onde a planta poderá tolerar concentrações baixas de aleloquímicos, não influenciando em seu crescimento.

Observa-se, na Figura 3, um aumento no percentual de germinação de sementes de sorgo até a concentração de $2,96 \%$ (p/v), proporcionando germinação de $84,49 \%$, a partir daí a germinação tende a diminuir com o aumento da concentração do extrato, seguindo tendência quadrática. Guenzi \& Mccalla (1966), relatam que os efeitos de aleloquímicos podem ser benéficos para a germinação, principalmente quando aplicados em quantidades muito pequenas.

Quanto ao índice de velocidade de germinação, para a alface (Figura 4), observa-se que os maiores percentuais de redução ocorreram com o extrator inorgânico (água), com médias de redução de 12,74\%; 52,77\% e 74,98\%, quando comparados ao tratamento testemunha $(0 \% \mathrm{p} / \mathrm{v})$, para as concentrações de 2,$5 ; 5$ e $10 \%$, respectivamente.

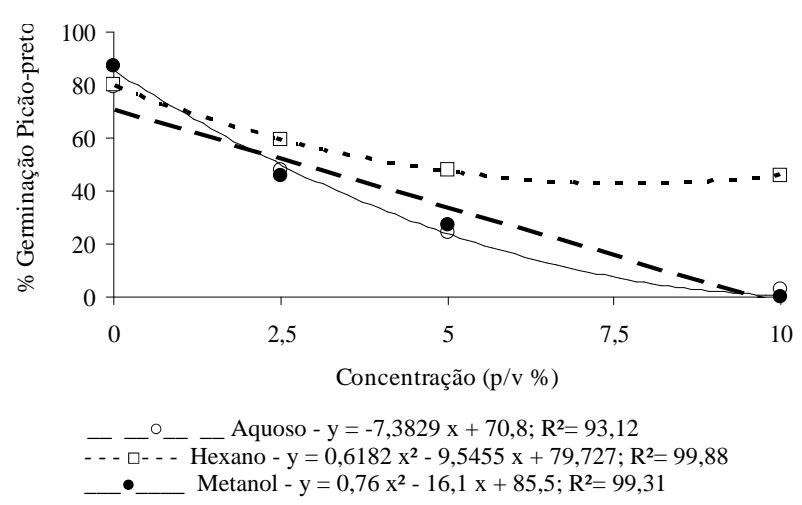

Figura 2 - Percentual de germinação de plântulas de picãopreto, submetidas à concentrações de extratos de nim (p/ v), utilizando água destilada, hexano e metanol como extratores. UFLA, Lavras - MG, 2007 


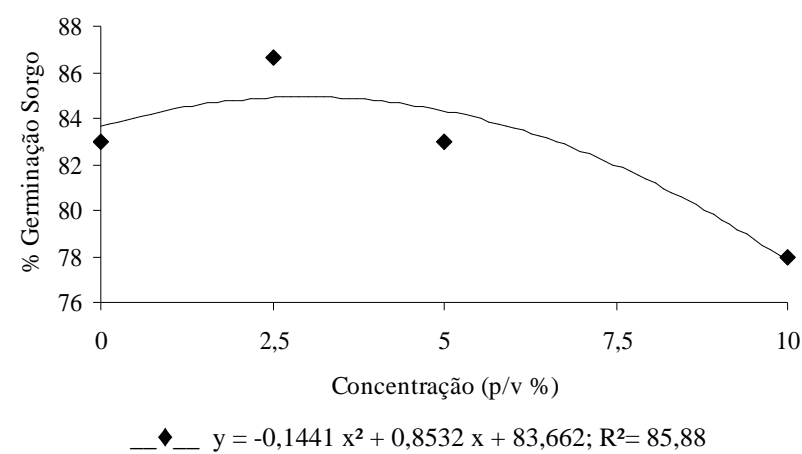

Figura 3 - Percentual de germinação de plântulas de sorgo, submetidas à concentrações de nim (p/v). UFLA, Lavras MG, 2007.

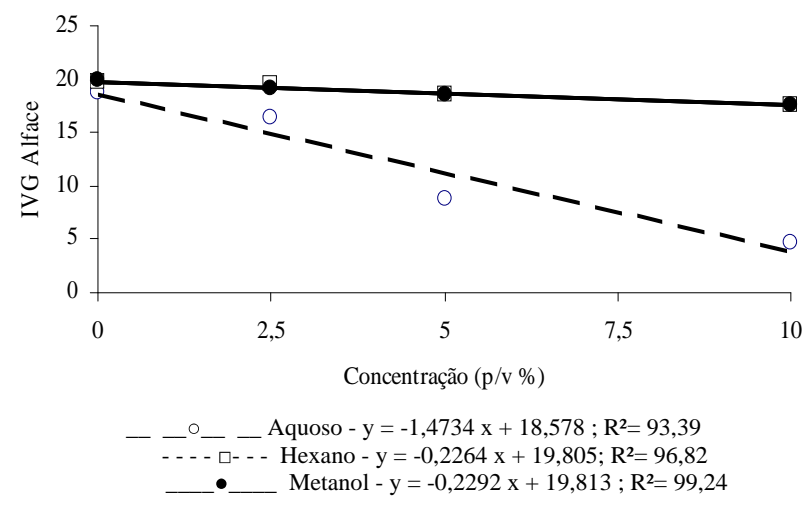

Figura 4 -Índice de velocidade de germinação de plântulas de alface, submetidas à concentrações de extratos de nim (p/v), utilizando água destilada, hexano e metanol como extratores. UFLA, Lavras - MG, 2007.

De acordo com a Figura 5, com o aumento da concentração, todos os extratores reduziram o índice de velocidade de germinação para o picão-preto, obedecendo tendência quadrática de redução. Para a concentração de $5 \%(\mathrm{p} / \mathrm{v})$, houve redução no índice de velocidade de germinação de $80,10 \% ; 65,77 \%$ e $73,13 \%$, quando comparados com o tratamento testemunha $(0 \% \mathrm{p} / \mathrm{v})$, para os extratores água destilada, hexano e metanol, respectivamente.

De acordo com a Figura 6, pode-se verificar que independente da concentração utilizada, o extrato aquoso foi o mais prejudicial ao índice de velocidade de germinação de sementes de sorgo, se diferenciando dos demais extratores. Inderjit \& Dakshini (1996) citam que a utilização de solventes inorgânicos, como clorofórmio, éter, hexano e metanol, devem ser evitadas por não ocorrer em condições naturais, podendo liberar compostos que não atuariam alelopaticamente.

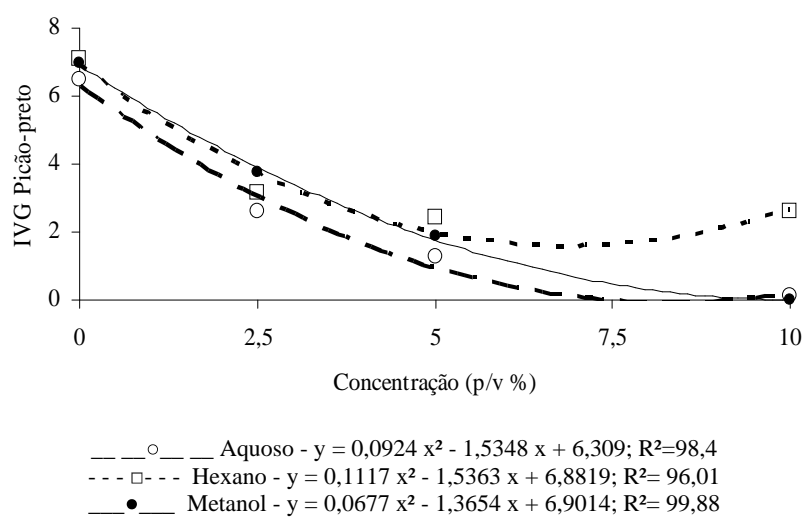

Figura 5 - Índice de velocidade de germinação de plântulas de picão-preto, submetidas à concentrações de extratos de nim (p/v), utilizando água destilada, hexano e metanol como extratores. UFLA, Lavras - MG, 2007.

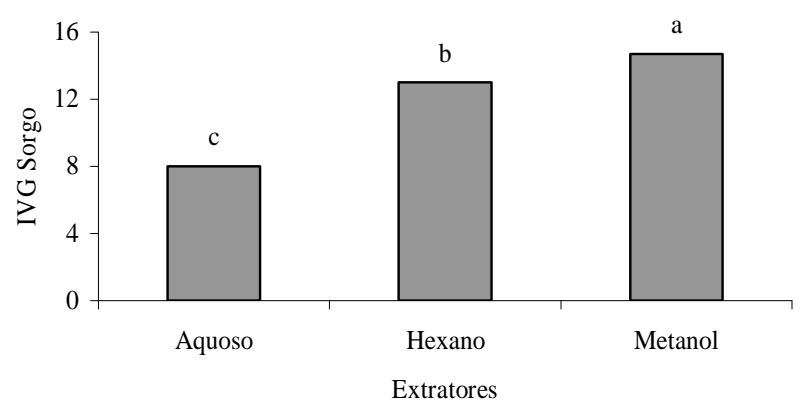

Colunas não seguidas pela mesma letra diferem significamente entre si pelo teste Scott-Knott, a 5\% de probabilidade.

Figura 6 - Índice de velocidade de germinação de plântulas de sorgo submetidas a extratos de nim utilizando água destilada, metanol e hexano como extratores. UFLA, Lavras - MG, 2007

Quando se analisou o acúmulo de biomassa para as espécies de picão-preto e sorgo (Figuras 8 e 9), para todos os extratos utilizados, pode-se verificar que o acúmulo de biomassa seca foi inversamente proporcional à concentração do extrato de nim aplicado. Observou-se na Figura 8, que quando a planta teste foi o picão-preto, o extrato metanólico foi o que promoveu menor acúmulo de biomassa seca, indicando que a forma de extração tem potencial extremamente tóxico para esta espécie. Para o sorgo, (Figura 9), o extrato hexanólico foi o que promoveu menor acúmulo de biomassa, com médias de 57,7; 40,06 e $27,76 \mathrm{mg}$ para as concentrações de 2,5; 5 e $10 \% \mathrm{p} / \mathrm{v}$, respectivamente. 


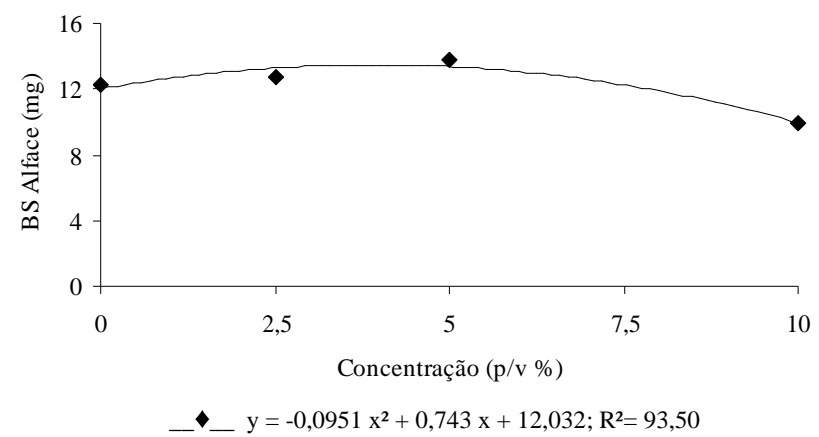

Figura 7 - Biomassa seca acumulada de plântulas de alface submetidas à concentrações de nim (p/v). UFLA, Lavras MG, 2007.

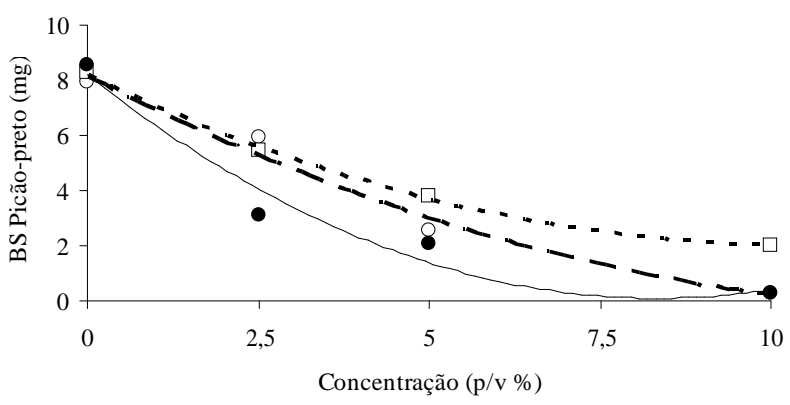

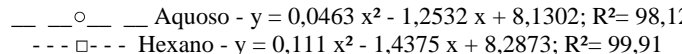

_-_ Metanol - $\mathrm{y}=0,1172 \mathrm{x}^{2}-1,9497 \mathrm{x}+8,2005 ; \mathrm{R}^{2}=95,84$

Figura 8-Biomassa seca acumulada de plântulas de picãopreto, submetidas à concentrações $(\mathrm{p} / \mathrm{v})$ de extratos de nim, utilizando água destilada, hexano e metanol como extratores. UFLA, Lavras - MG, 2007.

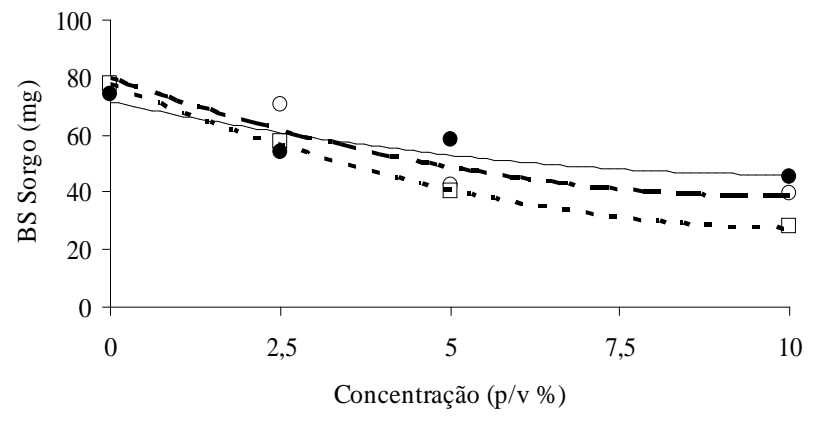

- - - - A Aquoso $-\mathrm{y}=0,4176 \mathrm{x}^{2}-8,2936 \mathrm{x}+79,832 ; \mathrm{R}^{2}=88,38$ - Metanol - $\mathrm{y}=0,2354 \mathrm{x}^{2}-4,9029 \mathrm{x}+71,51 ; \mathrm{R}^{2}=82,13$

Figura 9 - Biomassa seca acumulada de plântulas de sorgo, submetidas à concentrações (p/v) de extratos de nim, utilizando água destilada, hexano e metanol como extratores. UFLA, Lavras - MG, 2007.

\section{CONCLUSÕES}

Extratos aquosos, metanólicos e hexanólicos de nim (Azadirachta indica A. Juss.) exercem efeitos negativos acentuados no percentual de germinação e índice de velocidade de germinação sobre plântulas de sorgo, alface e picão-preto.

O extrato aquoso de folhas de nim mostrou-se com maiores potencialidades alelopáticas, com relação a efeitos detrimentais sobre o índice de velocidade de germinação nas espécies estudadas e sobre maiores efeitos detrimentais no percentual de germinação de alface.

\section{REFERÊNCIAS BIBLIOGRÁFICAS}

AERTS, R. J.; MORDUE, J. Feeding deterrence and toxicity of neem triperpenoids. Journal Chemical Ecology, New York, v. 23, n. 9, p. 2117-2132, 1997.

BRASIL. Ministério da Agricultura e da Reforma Agrária. Regras para análise de sementes. Brasília, DF: SNAD/ DNDV/CLAV, 1992. 365 p.

BLUM, U. Designing laboratory plant debris-soil biossays: some reflections. In: INDERJIT; DAKSHINI, K. M. M.; FOY, C. L. (Eds.). Principles and practices in plant ecology. Boca Raton: CRC, 1999. p. 17-23.

CARVALHO, G. J.; FONTANETTI, A.; CANÇADO, C. T. Potencial alelopático do feijão de porco (Canavalia ensiformes) e da mucuna preta (Stilozobium aterrimum) no controle da tiririca (Cyperus rotundus). Ciência e Agrotecnologia, Lavras, v. 26, n. 3, p. 647-651, mar./abr. 2002.

FERREIRA, A. G.; AQUILA, M. E. A. Alelopatia: uma área emergente da ecofisiologia vegetal. Revista Brasileira de Fisiologia Vegetal, Londrina, v. 12, n. 1, p. 175-204, 2000 .

GOMIDE, M. B. Potencialidades alelopáticas dos restos culturais de dois cultivares de cana-de-açúcar (Saccharum sp), no controle de algumas plantas daninhas. 1993. $96 \mathrm{f}$. Tese (Doutorado em Fitotecnia) - Escola Superior de Agricultura Luiz de Queiroz, Universidade de São Paulo, Piracicaba, 1993

GUENZI, W. D.; McCALLA, T. M. Phytotoxic substances extract from soil. Soil Science Society America Proceedings, Madison, v. 30, n. 3, p. 214-216, May/June 1966. 
INDERJIT; DAKSHINI, K. M. M. On laboratory biossays in allelopathy. The Botanical Review, New York, v. 61, n. 1, p. 28-44, Jan./Mar. 1996.

LABOURIAU, L. G. A germinação das sementes. Washington, DC: OEA, 1983.

LORENZI, H. Manual de identificação e controle de plantas daninhas: plantio direto e convencional. 4. ed. Nova Odessa: Plantarum, 2000. 383 p.

MAGUIRE, J. D. Speed of germination in selecting and evaluating for seedling emergence and vigor. Crop Science, Madson, v. 1, n. 1, p. 176-177, 1962.

MAREDIA, K. M.; SEGURA, O. L.; MIHM, J. A. Effects of neem, Azadirachta indica, on six species of maize insect pests. Tropical Pest Management, London, v. 38, p. 190195, 1992.

MEDEIROS, A. R. Determinação de potencialidade alelopáticas em agroecossistemas. 1989. 92 f. Tese (Doutorado em Agronomia) - Escola Superior de Agricultura Luiz de Queiroz, Piracicaba, 1989.

NIEMEYER, H. M. Hydroxamic acids (4-hydroxy-1,4benzoxazin-3-ones), defense chemicals in the gramineae. Phytochemistry, Oxford, v. 27, n. 11, p. 33493358, Nov. 1988.
PRATLEY, J. E.; NA, M.; HAIG, T. Following a specific protocol establish allelopathy conclusively: an Australian case study. In: MACIAS, F. A.; GALINDO, J. C. G.; MOLINILLO, J. M. G.; CUTLER, H. G. (Eds.). Recent advances in allelopathy. Cadiz: University of Cadiz, 1999. v. 1, p. 63-70.

SANTOS, C. C.; OLIVEIRA, D. F.; ALVES, L. W. R.; SOUZA, I. F.; FURTADO, D. A. S. Efeito de extratos orgânicos, associados ao surfactante TWEEN 80, na germinação e crescimento de plântulas de alface. Ciência e Agrotecnologia, Lavras, v. 28, n. 2, p. 296-299, mar./abr. 2004.

SCHMUTTERER, H. Properties and potential of natural pesticides from the neem

tree, Azadirachta indica. Annual Review of Entomology, Palo Alto, v. 35, p. 271-297, 1990.

SINDHU, A.; KUMAR, S.; SINDHU, G.; ALI, H.; ABDULLA, M. K. Effect of Neem (Azadirachta indica A. Juss) leachates on germination and seedling growth of weeds. Journal Allelopathy, New Delhi, v. 16, n. 2, p. 329$334,2005$.

SOUZA, C. N.; SOUZA, I. F.; PASQUAL, M. Extração e ação de sorgoleone sobre o crescimento de plantas. Ciência e Agrotecnologia, Lavras, v. 23, n. 2, p. 331-338, abr./jun. 1999. 